\title{
EFEITO DO EXPURGO COM FOSFINA (GASTOXIN) SOBRE A QUALIDADE FISIOLÓGICA DE SEMENTES DE MILHO E SORGO
}

\author{
RAMIRO VILELA ANDRADE ${ }^{1}$ e TELMA FALLIERI NASCIMENTO ${ }^{2}$
}

RESUMO. O Fosfeto de alumínio (Gastoxin) vem sendo cada vez mais utilizado no controle de pragas de grãos armazenados no Brasil. No entanto, ainda não é conhecida a ação deste inseticida fumigante sobre a qualidade fisiológica de sementes. Com este objetivo, foi realizado um experimento com sementes de milho (Zea mays L.) e sorgo (Sorghum bicolor (L.) Moench). Utilizaram-se duas cultivares de milho (BR 105 e Maia XVII) e três de sorgo (BR 005, BR 500 e BR 505) que sofreram de um a três expurgos em duas dosagens (dosagem recomendada pelo fabricante - 5 comprimidos de Gastoxin por $\mathrm{m}^{3}$ e dobro desta dosagem). Os resultados mostraram que as cultivares de milho e sorgo possuiam características distintas quanto à qualidade fisiológica, com sementes apresentando alto, médio e baixo vigor. $\mathrm{O}$ uso de inseticidas fumigante, $\mathrm{Al} \mathrm{PH}_{3}$ (Gastoxin), até o dobro da dosagem recomendada e até três expurgos, não causou variações significativas no poder germinativo e nos três testes de vigor aplicados às sementes destas espécies. Baseado nestes resultados, o Gastoxin pode ser utilizado no expurgo de sementes de milho e de sorgo, observadas as condições em que o experimento foi conduzido.

Termos para indexação:fumigação, fosfeto de alumínio, germinação, vigor, armazenamento.

\section{EFFECT OF PHOSPHINE FUMIGATION UPON \\ MAIZE AND SORGHUM SEED QUALITY}

\begin{abstract}
Aluminum phosphide (Gastoxin) has been successfuly used in Brazil to control storage cerals grain insects. However it is little known, the effect of this fumigant insecticide upon the seed quality of cultivars of corn and sorghum. Two cultivars of maize ( $\mathrm{Br}$ 105, Maya XVII) and three cultivars of sorghum (BR 005,

1 Engọ Agrọ MS, Pesquisador do Centro Nacional de Pesquisa de Milho e Sorgo EMBRAPA, Sete Lagoas-MG.
\end{abstract}

2 Engọ Agrọ Estagiária, CNPq - Centro Nacional de Pesquisa de Milho e Sorgo EMBRAPA, Sete Lagoas-MG. 
BR 500, BR 505) were fumigated with Gastoxin, one, two and three times using two dosage levels, (five and ten pellets for cubic meter). The results indicated that different levels of vigor obtained in the experiment were not affected by Gastoxin applications up to the double and three times the recommended comercial dosage.

Index terms: fumigation, aluminium phosphide $\left(\mathrm{Al} \mathrm{PH}_{3}\right)$, germination, vigor and seed storage.

\section{INTRODUÇÃO}

A danificação por insetos, em sementes de milho (Zea mays L.) e de sorgo (Sorghum bicolor (L.) Moench), durante o armazenamento, tem causado um sério problema na sua qualidade fisiológica, afetando a germinação e o vigor, tornando-as imprestáveis para o plantio e mesmo para o consumo.

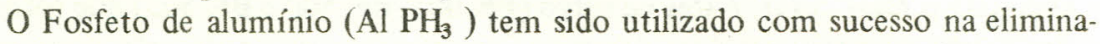
ção de insetos em grãos e sementes armazenadas de diversas espécies de cereais, legumes e gramíneas forrageiras. Foi estudada a tolerância de sementes de cereais, (Lindgren et al. 1958); pequenos legumes (Strong \& Lindgren 1963; Ahmad (1976) e de gramíneas forrageiras (White \& Jacobson 1972) ao Fosfeto de Alumínio e não foi constatado nenhum efeito prejudicial à qualidade fisiológica (germinação e vigor) das sementes destas espécies, mesmo quando apresentavam um elevado teor de umidade de 15 a 18\% (Gelosi et al. 1981). Por outro lado, sementes de sorgo, quando submetidas ao tratamento com brometo de metila, acusaram uma queda nos índices de germinação e vigor e, o seu grau de sensibilidade foi maior com o aumento da dosagem, teor de umidade das sementes, temperatura e tempo de duração do tratamento (Strong \& Lindgren 1963). As sementes de trigo, quando submetidas ao

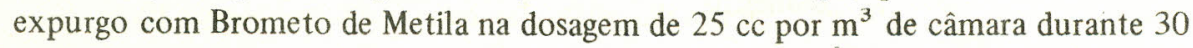
horas, não só retardaram sua germinação, como também tiveram diminuido seu poder germinativo cerca de 40 a $60 \%$, dependendo da variedade (Redaelli \& Cruz, 1960).

O Fosfeto de alumínio (Fosfina) vem sendo largamente empregado no Brasil, no controle de insetos em grãos e sementes armazenados com o nome comercial Gastoxin. No entanto, ainda não se conhece a sua atuação sobre a qualidade fisiológica de sementes.

Com o objetivo de avalirar o efeito do Gastoxin sobre a qualidade fisiológica das sementes de milho e sorgo, foi realizado o presente trabalho.

\section{MATERIAL E MÉTODOS}

O trabalho foi realizado no Laboratório de Análise de Sementes do Centro 
Nacional de Pesquisa de Milho e Sorgo. (CNPMS), em Sete Lagoas, MG.

O experimento constou da utilização de três cultivares de sorgo (BR 500, BR 005, BR 505) e de duas cultivares de milho (Maia XVII e BR 105), todas elas desenvolvidas pela EMBRAPA, com exceção do Maia XVII.

$\mathrm{O}$ material foi beneficiado e classificado em uma classificadora Clipper, modelo experimental, para uma melhor uniformidade no tamanho da semente. Para milho, utilizaram-se sementes que ficaram retidas na peneira de número $22(9,00$ $\mathrm{mm})$, e para sorgo, sementes retidas na peneira de número $1 / 14 \times 1 / 4(1,80 \times 6,00$ $\mathrm{mm})$.

O delineamento experimental utilizado foi o inteiramente casualizado sendo que os tratamentos foram dispostos no esquema fatorial, com cinco variedades, três expurgos, duas dosagens de Fosfina (produto comercial de Gastoxin) e uma testemunha (sem tratamento).

As dosagens foram as seguintes:

a) Dosagem recomendada pelo fabricante -5 comprimidos de Gastoxin por $\mathrm{m}^{3}$

b) Dobro da dosagem recomendada - 10 comprimidos de Gastoxin por $\mathrm{m}^{3}$

c) Testemunha (sem nenhum tratamento)

Os expurgos foram realizados em tambores de 200 litros, adapatados de maneira a evitar vazamentos do gás, onde as sementes foram tratadas durante 48 horas, acondicionadas em sacolas de pano.

Utilizaram-se quatro amostras para cada variedade e, no início do experimento, três receberam tratamento com Fosfina, e a outra utilizada como testemunha. Todas elas foram armazenadas por seis meses, em condições ambientais do Laboratório de Análise de Sementes do Centro Nacional de Pesquisa de Milho e Sorgo. Trinta dias após a primeira fumigação, duas das três amostras tratadas foram fumigadas pela segunda vez, e armazenadas. Trinta dias após a segunda fumigação, uma das duas partes tratadas passou por uma terceira fumigação.

Desta maneira, tivemos sementes que não receberam tratamento, (testemunha) sementes tratadas uma só vez no início do experimento, duas e três vezes no primeiro e segundo meses de armazenamento.

Todas as vezes em que as sementes receberam tratamento, tomou-se uma amostra para realização de teste de germinação, com a finalidade de comparar o comportamento das sementes tratadas com a testemunha. No final do experimento, após seis meses de armazenamento, além do teste de germinação, foram realizados os testes de envelhecimento precoce e índice de emergência de acordo com BRASIL. Ministério da Agricultura, (1976); Hoppe (1956); Zink (1970) e Maguire (1962), respectivamente. 


\section{RESULTADOS E DISCUSSÃO}

No início do experimento, determinaram-se os teores de umidade das sementes em um aparelho universal e os resultados foram os seguintes: BR $005-10,3 \%$; BR 500 - 11,5\%; BR 505 - 11,7\%; Maia XVII - 13,9\% e BR $105-14,2 \%$.

Os resultados alcançados encontram-se resumidos nas Tabelas 1 e 2, respectivamente, para as cultivares de milho e sorgo e, nas Figs. 1 e 2. Pode-se observar que o poder germinativo e o vigor (testes de Hoppe, envelhecimento precoce e índice de emergência) das sementes destas duas culturas não sofreram alteração, após seis meses de armazenamento, quando submetidas ao tratamento com fosfina (Gastoxin), nas duas dosagens utilizadas e até três expurgos (Fig. 2).

Tabela 1. Quadrados módios (QM), ágnificância estatística (F) e coeficientes de variaçlo obtidos na análise de variância dos testeade germinaço e vigor (Hoppe, Envelhecimento Precoce-EP e Indice de Emergencia-IE) para as cultivares de milho (BR 105 e Maia XVII).

\begin{tabular}{|c|c|c|c|c|c|c|c|c|}
\hline \multirow{2}{*}{$\begin{array}{l}\text { Causas de } \\
\text { Variaçāo }\end{array}$} & \multicolumn{2}{|c|}{ Germinação } & \multicolumn{2}{|c|}{ Hoppe } & \multicolumn{2}{|c|}{ E.P. } & \multicolumn{2}{|c|}{ I.E. } \\
\hline & QM & $\mathbf{F}$ & $\mathrm{QM}$ & $F$ & QM & $\mathbf{F}$ & QM & $\mathrm{F}$ \\
\hline \multicolumn{9}{|l|}{ (T ratamentos) } \\
\hline Variedade (V) & 108,00 & $33,28^{*}$ & 16,33 & NS & 102,08 & $75,34 *$ & 0,08 & NS \\
\hline Dosagens (D) & 0,34 & NS & 0,00 & NS & 14,08 & NS & 0,06 & NS \\
\hline Expurgo (E) & 1,09 & NS & 0,59 & NS & 16,00 & NS & 0,18 & NS \\
\hline Entre Testemunhas & 220,50 & $67,95 *$ & 2,00 & NS & 4,50 & NS & 0,11 & NS \\
\hline $\mathrm{V} \times \mathrm{D}$ & 12,00 & NS & 0,34 & NS & 6,76 & NS & 0,12 & NS \\
\hline$V \times E$ & 4,75 & NS & 5,09 & NS & 16,98 & NS & 0,10 & NS \\
\hline$D \times E$ & 0,58 & NS & 3,25 & NS & 4,34 & NS & 0,02 & NS \\
\hline Test. x Resto & 119,05 & $36,69^{*}$ & 3,05 & NS & 0,11 & NS & 0,04 & NS \\
\hline V x D x E (Resíduo) & 3,25 & & 3,08 & & 1,36 & & 0,04 & \\
\hline Coef. Variaçāo (\%) & \multicolumn{2}{|c|}{1,93} & \multicolumn{2}{|c|}{1,90} & \multicolumn{2}{|c|}{1,30} & \multicolumn{2}{|c|}{2,21} \\
\hline
\end{tabular}

Tabela 2. Quadrados médios (QM), rignificância estatística (F) e coeficiente de variaçâo obtidos na análise de variância dos testes de germinação e vigor (Hoppe, Envelhecimento precoce-EP e Índice de emergência-IE) para as cultivares de songo (BR 500, BR 505 e BR 005).

\begin{tabular}{|c|c|c|c|c|c|c|c|c|}
\hline \multirow{2}{*}{$\begin{array}{l}\text { Causas de } \\
\text { Variação }\end{array}$} & \multicolumn{2}{|c|}{ Germinação } & \multicolumn{2}{|c|}{ Hoppe } & \multicolumn{2}{|c|}{ E.P. } & \multicolumn{2}{|c|}{ I.E. } \\
\hline & $\mathrm{QM}$ & $F$ & QM & $\mathrm{F}$ & QM & $\mathrm{F}$ & QM & $\mathrm{F}$ \\
\hline \multicolumn{9}{|l|}{ Tratamentos } \\
\hline Variedades (V) & 108,00 & $2061,27 * *$ & 16,33 & $488,01 *$ & 102,08 & $252,19 *$ & 0,08 & $601,00 *$ \\
\hline Dosagens (D) & 0,34 & NS & 0,00 & NS & 14,08 & NS & 0,06 & NS \\
\hline Expurgo (E) & 1,09 & NS & 0,59 & NS & 16,00 & NS & 0,18 & NS \\
\hline Entre Testemunhas & 220,50 & $442,06^{* *}$ & 2,00 & $87,65^{* *}$ & 4,50 & $50,47 * *$ & 0,11 & $103,75^{* 4}$ \\
\hline $\mathrm{V} \times \mathrm{D}$ & 12,00 & NS & 0,34 & NS & 6,76 & NS & 0,12 & NS \\
\hline$V \times E$ & 4,75 & NS & 5,09 & NS & 16,98 & NS & 0,10 & NS \\
\hline $\mathrm{D} \times \mathrm{E}$ & 0,58 & NS & 3,25 & NS & 4,34 & NS & 0,02 & NS \\
\hline Test. $x$ Resto & 119.05 & $46,15^{* *}$ & 3,05 & NS & 0,11 & NS & 0,04 & NS \\
\hline V $\times$ D $\times E$ (Resíduo) & 3,25 & & 3,08 & & 1,36 & & 0,04 & \\
\hline Coef. Variação (\%) & \multicolumn{2}{|c|}{1,90} & \multicolumn{2}{|c|}{7,89} & \multicolumn{2}{|c|}{9,59} & \multicolumn{2}{|c|}{3,78} \\
\hline
\end{tabular}




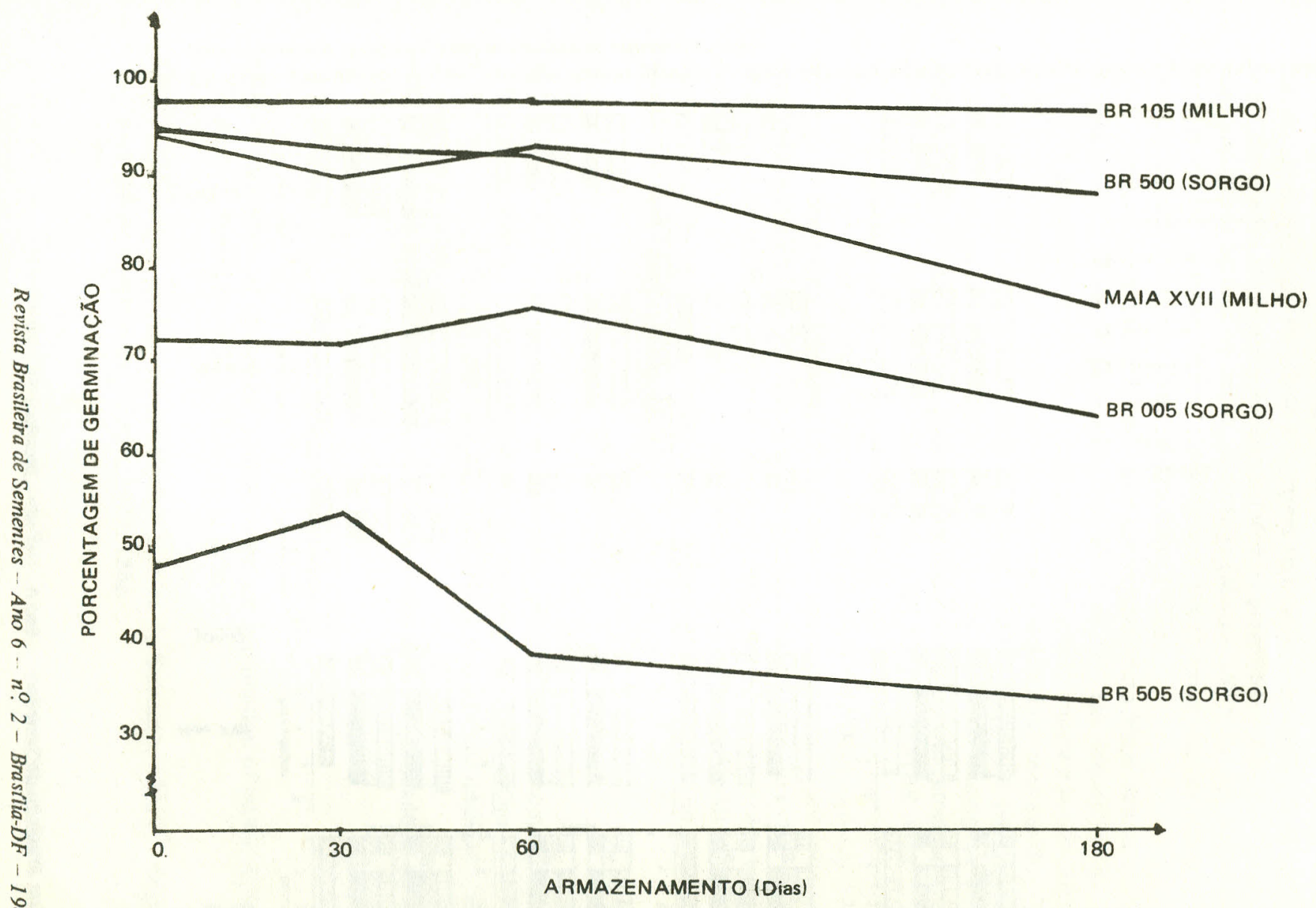

Fig. 1. Porcentagem de germinação das testemunhas das cultivares de milho e sorgo armazenadas em condições ambientais durante seis meses. 


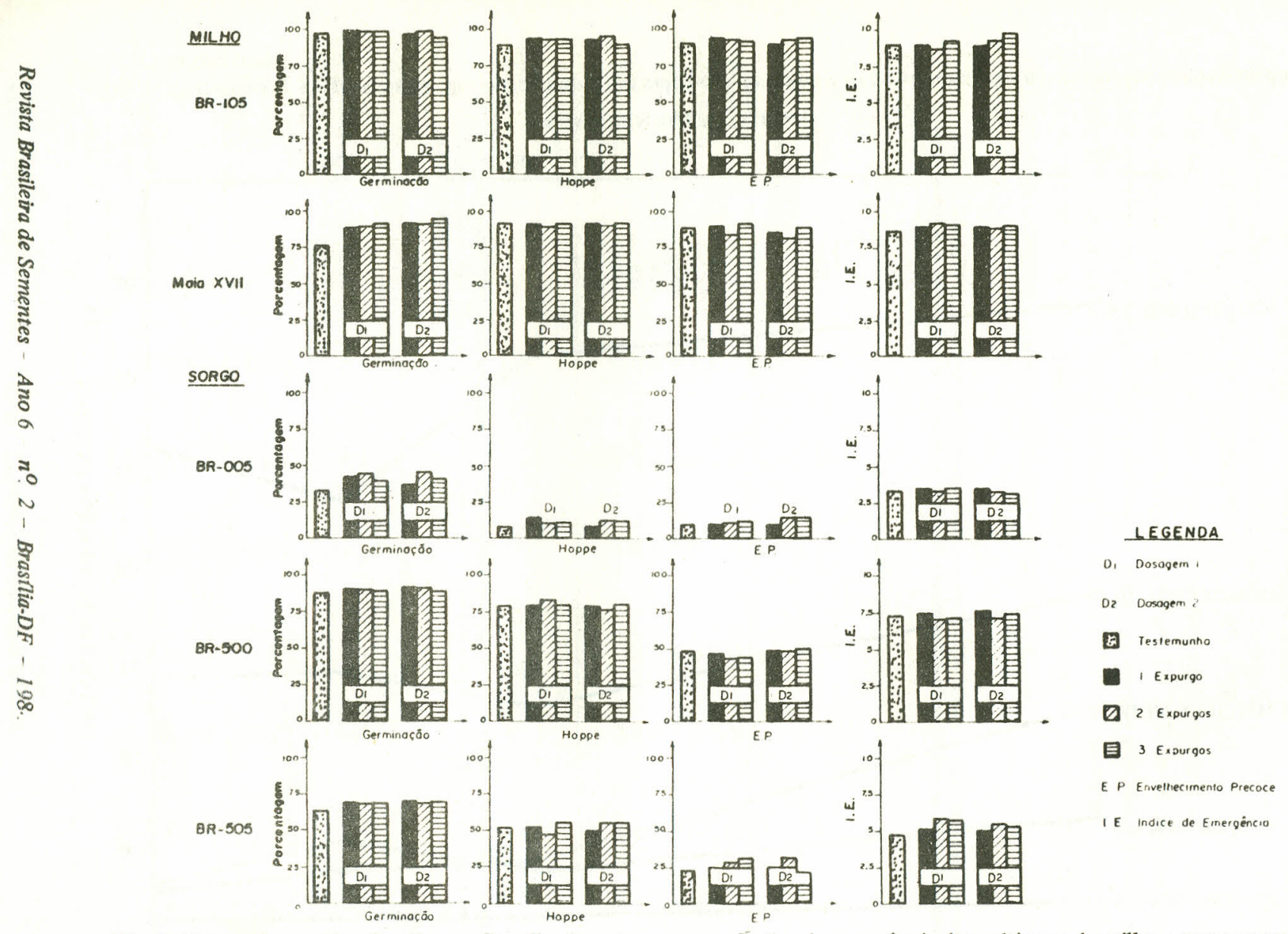

Fig. 2. Têtes de germinação, Hoppe, Envelhecimento precoce e Indice de emergência das cultivares de milho e sorgo expurgadas com o Gastoxin (Fosfina), após seis meses de armazenamento. 
As análises de variância aplicadas aos testes de germinação e vigor (Tabelas 1 e 2), ñ̃o apresentram diferenças significativas nos testes de $F$, para as seguintes variáveis: dosagem, expurgo, variedade $\mathrm{x}$ dosagem variedade $\mathrm{x}$ expurgo e dosagem $\mathrm{x}$ expurgo. Resultados semelhantes aos encontrados no presente trabalho, foram constatados por outros autores em sementes de trigo (Monro 1962; Zutshi 1966 e Lindgren et al. 1958) milho (Puzzi, 1963; Comes 1964), sorgo (Strong \& Lindgren 1963), gramíneas forrageiras (White \& Jacobson 1972).

Observando-se a Tabela 1, verifica-se que o tratamento Variedade para o mitho apresentou diferenças significativas nos testes de " $F$ " nas determinações dos índices de germinação e envelhecimento precoce, enquanto que para as três cultivares de sorgo estudadas, o tratamento Variedade apresentou diferenças significativas para todos os testes aplicados (Tabela 2). Isto nos leva a conclusão de que estas cultivares possuiam características distintas quanto a suas qualidades fisiológicas, resultando em lotes de sementes que poderiam ser classificados em três categorias de vigor: alto (BR 105 e Maya XVII) médio (BR 500 e BR 505) e baixo (BR 005).

Foi realizado um estudo de armazenamento com as testemunhas destas cultivares, para determinar os seus potenciais de armazenamento em condições ambientais, durante seis meses. A cultivar BR 005 apresentou uma queda no seu poder germinativo a partir do segundo mês (Fig. 1). A causa deste declínio no índice de germinação foi atribuída ao baixo vigor destas sementes, acusado nos testes de Hoppe, envelhecimento precoce e índice de emergência (Fig. 2). Para as demais cultivares, o armazenamento por seis meses não ocasionou uma queda acentuada no poder germinativo de suas sementes (Fig. 1).

\section{CONCLUSOES}

- A fosfina ( $\mathrm{Al} \mathrm{PH}_{3}$ ), comercializada através do produto de nome comercial Gastoxin, não afetou a qualidade fisiológica das sementes de sorgo e milho.

- Até um número de três expurgos, não se constatou alterações na qualidade fisiológica da semente.

- Uma dosagem superior à recomendada (dobro) não afetou a qualidade da semente.

\section{REFERENCIAS}

AHMAD, M. Effect of phosphine fumigation on the germination of edible legume seeds. J. Stored Prod. Res., (12):211-2, 1976.

BRASIL. Ministério da Agricultura. Equipe Técnica de Sementes e Mudas. Regras para análise de sémentes. Brasília, 1976. 188p. 
CORNES, M.A. An experimental to compare the effects of carbon Tetrachloride and Phostoxin on the germination and subsequente growth of maize. s.1., N.S.P.R.I Annual Report, 1964. (Technical Report, 14).

GELOSI, A.; LOVATO, A. \& MONTANARI, M. Effetti di fumigazioni con fosfina sulla vitalità dei semi di cereali. Sementi Elette, 27(6):19-21, 1981.

HOPPE, P.E. Cold testing. In: HYBRID CORN INDUSTRY RESEARCH CONFERENCE, 11. Proceedings... s.1., 1956. p.68-74.

LINDGREN, D.L.; VINCENT, L.E. \& STRONG, R.G. Studies on hidrogen phosphide as a fumigant. J. Econ. Entomol., Maryland, 51(6):900-3, Dec. 1958.

MAGUIRE, J.D. Speed of germination aid in selection and evaluation for seedling emergence and vigor. Crop Sci., Madison, 2(2):176-7, 1962.

MONRO, H.A.V. Manual de fumigación contra insectos. Roma, FAO, 1962. 323p.

PUZZI, D. Conservação de grãos armazenados. São Paulo, Agronômica Ceres, 1963 $217 \mathrm{p}$.

RADAELLI, D.C. \& CRUZ, F.Z. da. Efeitos do expurgo com brometo de metila sobre a germinação do trigo. Agron. Sulriogr., 5(1):61-4, 1960.

STRONG, R.G. \& LINDGREN, D.L. Germination of grain sorghum and sudan grass seeds after fumigation with methyl bromide and dydrocyanic acid. J. Econ. Entomol., 56(2):144-9, 1963.

WHITE, G.D. \& JACOBSON, E.T. Phosphine fumigation. Effects on the germination of grass seed. J. Econ. Entomol., 65(5):1523-4, 1972.

ZINK, E. Vigor de sementes de milho. In: SEMINARIO BRASILEIRO DE SEMENTES, 2, Pelotas, 1968. Anais... Rio de Janeiro, IPEAS/SIA, 1970. p.231-2.

ZUTSHI, M.K. Effect of Aluminium phosphide (phostoxin) on de germination of certain ceral and vegetable seeds. Bull grain technol., 4(4):197-9, 1966. 